\title{
A theoretical study of the stability of disulfide bridges in various b-sheet structures of protein segment models
}

Natalie J. Galant, Heeyeon Cheryl Song, Imre Jákli, Bela Viskolcz, Imre G. Csizmadia, Svend J. Knak Jensen, Andras Perczel

\section{Introduction}

\subsection{Amyloid formation and hydrogen bonding}

Amyloid fibrils are associated with a number of human diseases, including Alzheimer's disease, Huntington's disease, Type II Diabetes Mellitus, and some prion diseases such as spongiform encephalopathies [1,2]. Despite the different protein precursors for each disease, all of them seemed to share a common structural characteristic: the 'cross-b spine' [3]. Much effort had been successful to realize the structure of such spine, the driving force responsible for the formation of amyloid fibrils. In 2005, Eisenberg and his team postulated the structure of the cross b spine of amyloid-like fibrils and named the sheet-sheet interaction a 'steric zipper' [4]. During their studies they observed 11 hydrogen bonds responsible for the stability of $b$-sheets of their GNNQQNY model, a fibril-forming segment of yeast protein Sup35. Five of these were backbone $\mathrm{C}=\mathrm{O}---\mathrm{H}-\mathrm{N}$ hydrogen bonds, while the remaining six were formed by amide-amide or amide-hydroxyl hydrogen bonds between residue side chains.

Recent studies have also concluded that the strong backbone-backbone interactions (primarily interchain hydrogen bonds), that are well pronounced in $\beta$-strands are the driving force of amyloid-like nano-structure formation leading to macroscopic aggregation and thus to the 'dead-end' of protein folding [5]. The hydrogen/deuterium exchange of amide protons further illustrated that amyloid fibrils are stabilized by an extensive network of hydrogen bonds substantiating $\beta$-sheets [6]. Previous work on b-sheet models by using polypeptide strands, such as the (Ala)6 hexapeptide, have indicated that the anti-parallel topology is thermodynamically more favored to parallel configurations [7].

The present study aims to explore whether Cys residue pairs in polypeptide strands can stabilize (in the thermodynamic sense) $\beta$-sheets formation. A model containing two strands of chiral amino acid residues was designed. The model allows the possibility to form either a single or double inter- or intra-strand disulfide bridges (Figures 1 and 2) and to assess the stability of $\beta$ strand foldamers as function of disulfide bridges and their oxidation states, via a quantum mechanical based description, heading to explain the intrinsic feature of polypeptide aggregation.

\subsection{Disulfide bridges and aggregation pathways}

Several studies suggest that structural rearrangement of protein leading to aggregation is directly caused by improper disulfide bond formed either between or within proteins. Improper disulfide bridge formation is the main cause of aggregation for T4 lysozyme[8] and SOD1 (superoxide dismutase) [9]. Loss of disulfide bond also may be a mechanism to promote amyloid fibril formation in vitro for some proteins, such as Bence Jones proteins [10] and human prion proteins [11]. On the other hand, some reduction of disulfide bridges within a protein prevented formation of amyloidfibrils in certain proteins, such as insulin [12] and b2-microglobulin [13]. Reduced proteins instead tend to form filaments rather than fibrils. These findings together highlight the significance of disulfide bridges in amyloid fibril formation. 
Cross-strand pairing is considered as a significant contributor tob-sheet assembly and stability in globular proteins although certain cross-strand pairs bring stability to the protein, while some others do not [14-18]. The stability of the cross-strand pair also depends on if the Cys pair is directly hydrogen bonded (HB) or not hydrogen bonded (NHB).

Cys has an extraordinary high preference to be its own crossstrand pair partner, making Cystine or Cys-SS-Cys, both in the H-bonded and non-H-bonded sites [16]. Cystine is more often in NHB than in HB site of proteins [19]. The effect on stability of a cross-strand disulfide within proteins is an ongoing question [19] as disulfide bridges are rarely found between adjacent $\beta$ strands of proteins. This observation was even once ruled as a 'forbidden' structural scenario [20,21]. As more information on protein structures accumulate, so have the discovery of more and more cases, where cross-strand disulfides exist [19]. Disulfides also play a role in several other biochemical processes [22-28].

\section{Models used}

The penta-peptide model of (Ala-Cys-Ala-Cys-Ala) $)_{2}$ is investigated here, focusing on its stability as function of the topology and oxidation state of the Cys residues (Figure 1). Both the crossstrand $\left(\mathrm{S}_{\text {Inter, }}, \mathrm{S}_{\text {Inter }}\right)$ and intra-strand $\left(\mathrm{S}_{\text {Intra }}, \mathrm{S}_{\text {Intra }}\right)$ disulfide bridge topologies were tested, aiming to reveal their relative stability. Since the anti-parallel $b$-sheet structures are comprised of alternating smaller and larger rings, with 10 and 14 atoms, respectively, we use two different anti-parallel $\beta$-sheet notations throughout the Letter, $10_{3} 14_{2}$ and $14_{3} 10_{2}$, to indicate the topology of the pentapeptide. The subscripts within these notations indicate how many rings occur in each structure. For example, the $14_{3} 10_{2}$ notation describes a pentapeptide $\beta$-sheet which contains three 14-membered rings and two 10membered rings (Figure 1). All disulfide bridge topologies were embedded in the $14_{3} 10_{2}$ or $10_{3} 14_{2}$ anti-parallel, as well as parallel b-sheet backbone structures (Figure 2).

In proteins, anti-parallel $\beta$-sheets can be initiated either by a 14 - or a 10 -membered $\mathrm{H}$-bonded ring structure (Figure 1B), however, those 'starting' with a '14' subunit have been found to be more frequent as well as more energetically favorable of the two H-bonded structures [5,29]. Protein structures containing parallel $\beta$-sheets and their corresponding 12-membered rings, however, were found to be less thermodynamically stable and less frequent in comparison to either of the two antiparallel structures [28]. The current study aims to explore whether disulfide linkages cause any change in terms of the relative stabilities of these isomeric forms. The stability of the SS linkage is measured by free energy changes of red-ox reactions. In biological systems, usually mild oxidizing agents play a role and the Quinone/hydroquinone structural features occur in numerous bioactive molecules, such as coenzyme Q10, Vitamin K and Vitamin E. For this reason, we use the simple Quinone $(1,4-$ benzoquinone, also known as paraquinone) as a mild oxidizing agent to assess the stabilizing effect of disulfide linkage formation.

\section{Methods}

\subsection{Computations}

Anti-parallel and parallel models of $\{$ For-(L-Ala)5-NH2 2 were geometry optimized using GAUSSIAN09 [29]. Specific side chain rotamers of interest (Figure 2) were studied; considering the $\mathrm{g}+\left(60^{\circ}\right)$, a $\left(180^{\circ}\right)$ and g- $\left(300^{\circ}\right)$ conformers of the reduced Cys, the most stable conformers were selected. Assuming internal structure symmetry, only one partially oxidized structure was calculated for the $10_{3} 14_{2}$ and $14_{3} 10_{2}$ anti-parallel structures. For the Parallel model, calculations of the partially oxidized state included two different models. These included the $s b \_c$, in which the single disulfide 
bridge was formed towards the carboxy terminus, and the $s b \_n$, where the single bridge was formed towards the amino terminus of the $\beta$-strands of the parallel models.

\subsection{Levels of theory applied}

The majority of calculations were performed at the level B3LYP/6-31G(d) for practical reasons. There are few measured thermodynamic data available to assess the accuracy of the calculated thermodynamic data for gas phase reactions involving the S-S bond. As a simple benchmark we consider the reaction:

$2 \mathrm{SH}_{2} \rightarrow \mathrm{HSSH}+\mathrm{H}_{2}$

The calculated enthalpy difference $\left(\Delta \mathrm{H}^{0}\right)$ for $(1)$ is only $1.2 \mathrm{~kJ} /$ mol higher than the experimental value [30]. Calculations were also performed at two higher levels of theory [wB97xd/6-31G(d) to assess the importance of dispersion interactions and B3LYP/6-311G(d,p)] to evaluate basis set effects (Table $\mathrm{S} 1$ ). All thermodynamic functions were based on the vibration frequencies in the potential minima and calculated at a temperature of $25{ }^{\circ} \mathrm{C}$ and a pressure of one bar. The free energy changes on $\mathrm{S}-\mathrm{S}$ bond formation in aqueous solution were estimated from single point calculations using the polarized continuum model, IEFPCM.

\subsection{Disulfide formation reactions}

Formation of the disulfide bridge between two Cys residues resulting in a Cystine, may be written as:

2 Cys- $\mathrm{SH} \rightarrow \mathrm{Cys}-\mathrm{SS}-\mathrm{Cys}+\mathrm{H}_{2}$

Energetics associated with such a disulfide bridge formation was estimated via reaction (2) both for the partial and full oxidation of the reduced Ala-Cys-Ala-Cys-Ala pentapeptide in its $\beta$ strand form. All reaction pathways of the double disulfide formation starting from the reduced no-bridge (NB) AlaCys-Ala-Cys- Ala pentapeptide model (S0) via the partial-oxidized (S1) to the fully-oxidized (S2) forms, pathways I, II and III, are shown as thermodynamic cycles (Figure 3). To assess disulfide bridge strength, a pair of SH functional groups may be subjected to an oxidation reaction to generate an SS linkage. This can be achieved via direct oxidation using agents such as 1,4-benzoquinone (3) (Figure 3):

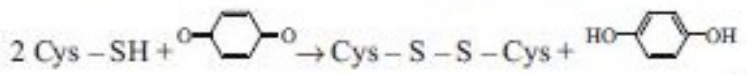

\subsection{Statistical analysis}

In order to explore the natural abundance of the X-Cys-X-Cys-X protein motif, a search was performed using the online version of the PDB (http://www.rcsb.org/pdb/search/advSearch.do) [31] using the following filtering criteria: Sequence Motif: XCXCX, Macromolecule type: protein chain but not any DNA or RNA or Hybrid,Homologue Removal cutoff at $40 \%$. This search resulted in 4468 proteins, which were downloaded and processed using the DSSPcont program [32]. The DSSPcont output was analyzed using in-house developed Java programs.

\section{Results and discussions}

\subsection{Statistical analysis of protein disulfide linkages}


By examining the 31813 records of the PDB search we found 592 of them were in b-strands. By further filtering the occurrences in b-strands, we found only two examples in which the cysteines were connected by an intra-strand disulfide linkage. Both of these examples occurred in glucosaminefructose-6-phosphate aminotransferase (3TBF) protein. We also examined the cases in which two XCXCX motifs were located in the different polypeptide chains of the same b-strand. Eight examples were found for that type of structure in two protein families: protegrin PG-1 (1PG1, 1ZY6), thetadefensines: BTD-2 (2LYE, 2M2G), HTD-2 (2LZI) and RTD-1(2LYF, 1HVZ). All of them contained inter-disulfide bridges between the two chains in the strand. Such bridges made the otherwise flexible small cyclic or turn-containing polypeptides more rigid.

\subsection{Structure characterization; hydrogen bond and SS networks}

Formation of a SS bridge will alter the local geometry and thus the length of the neighboring hydrogen bonds. The detailed variationof these bonds is given in Table S2. In each orientation, the NB model within each family of models was chosen as the reference structure to compare the effect of SSbond on the H-bonded network (Figure 4, Table S2). The gross features are as follows: A single SS inter-strand bridge leads to a shortening of up to $8 \%$ in the case of the anti-parallel orientations, with the $10_{3} 14_{2}$ orientation exhibiting the largest reduction. In the case of the parallel orientation the hydrogen bond length is modified by $\pm 1 \%$ only. An intra-strand SS bridge leads to a more complex variation with some hydrogen bonds getting longer. The adjustments are in the range $-10 \%$ to $8 \%$. The adjustment is smallest for the parallel orientation.Two inter-strand SS bridges lead to adjustments of the neighboring hydrogen bond lengths in the range of $-4 \%$ to $1 \%$. Again the adjustments are smaller for the $14_{3} 10_{2}$ and parallel orientations. Two intra-strand SS bridges in the sheets exhibit a variation in the range $-5 \%$ to $15 \%$ with the largest adjustment for the $10_{3} 14_{2}$ orientation and the smallest for the parallel orientation. Calculations at the two other levels of theory support this picture. Table S3 lists the bond length of the SS bridges for the various orientations of the two strands. It appears that the intra-strand SS bridges tend to be shorter than the inter-strand. However, the overall variation is small, in the range of $1-2 \%$.

\subsection{Extra stability due to disulfide bridge introduction}

\subsubsection{The single disulfide linkages}

At the B3LYP/6-31G(d) level the anti-parallel $14_{3} 10_{2}$ was found to be the most thermodynamically stable model amongst all three families, including each family's reduced (S0), partial (S1) and fully (S2) forms (Figure S1). In comparison to two single strands separated infinitely apart, all three models in their b-sheet configurations experienced a decrease in Gibbs free energy as result of hydrogen bond stabilization. Stability analysis completed by the partial oxidization reaction(Figure 3) shows that cross-strand disulfide bridge formation brought stabilization to b-sheets, in all three orientations, when using an oxidizing agent such as 1,4-benzoquinone (Figure 5). In most cases, the introduction of either an inter-strand or intrastrand disulfide bridge within the pentapeptide b-sheet resulted in structure stabilization (Table S5). The only case in which destabilization occurred was in the $10_{3} 14_{2}$ orientation, where the introduction of a single intra-strand disulfide bridge destabilizedthe b-sheet by $+9.2 \mathrm{~kJ} / \mathrm{mol}$ (Figure 5). Partial oxidation via single inter-strand disulfide introduction stabilized all three b-sheet families. Of the three models, the most thermodynamically stable configuration was the Parallel C-terminus model, which was $-23.7, \quad-1.4$ and $-24.6 \mathrm{~kJ} / \mathrm{mol}$ more stable than the anti-parallel $10_{3} 14_{2}$, and parallel N-terminus, anti-parallel $14_{3} 10_{2}$ configurations, respectively. In cases where a single intra-polypeptide disulfide bridge was introduced, the parallel 
orientation was once again the most thermodynamically favorable configuration, with it being -36.8 and -13.8 more stable than the anti-parallel $10_{3} 14_{2}$ and anti-parallel $14_{3} 10_{2}$ configurations, respectively. This data, therefore, demonstrates that the introduction of both interand intra-strand disulfide bridges often resulted in stabilization of the pentapeptide b-sheet models using the 1,4-benzoquinone oxidation mechanism (Figure 5, Table S4).

\subsubsection{Double disulfide linkages}

The introduction of two disulfide bridges, either inter- or intrastrand disulfides, resulted in stabilization of all b-sheet models (Figure 5). Of the fully oxidized models, following the introduction of two inter-strand disulfides, the most stabilized model was the Parallel orientation, with its free energy being -6.7 and $-31.7 \mathrm{~kJ} / \mathrm{mol}$ lower than the anti-parallel $10_{3} 14_{2}$ configuration andanti-parallel $14_{3} 10_{2}$ configurations, respectively. This result is somewhat surprising as previous investigations lead to a different picture [7]. In order to obtain some degree of assurance that the resul is not a peculiarity of the B3LYP/6-31G(d) method we have performed calculations at the two other theoretical levels mentionedin Section 3.2 as well as polarized continuum model single point calculations, mimicking an aqueous phase. The results of these calculations (Table S1) support that the parallel configuration is the most stable one, both in the gas phase and in aqueous solution. However, the difference in stability is in some cases not more than a few $\mathrm{kJ} / \mathrm{mol}$ suggesting that the local environment in a protein may have a decisive influence on which conformation is the stable one.

It is not surprising that the anti-parallel $14_{3} 10_{2}$ configuration shows the least stabilization following inter-strand di-oxidation $(+81.1 \mathrm{~kJ} / \mathrm{mol})$ because in comparison to two strands separatedinfinitely apart, its reduced form was the most stable of the three reduced models (Figure S1 and Table S5).

The parallel configuration was again the most thermodynamically stable of the three intrastrand di-oxidized models, being -14.4 and $-2.7 \mathrm{~kJ} / \mathrm{mol}$ more stable than the fully oxidized antiparallel1 $10_{3} 14_{2}$ models and anti-parallel $14_{3} 10_{2}$, respectively. It is clear from Figure 5 that increasing the number of intra-polypeptid disulfide bridges increases b-sheet stability, but that it is always more favorable than the introduction of a single intrastrand disulfide bridge, such as in the parallel model. It may be related to structural distortion following the introduction of two intra-strand disulfides in the parallel structure. Nevertheless, inall cases, it is always more favorable to introduce inter-strand disulfides rather than intra-strand disulfides within $\beta$-sheets.

\section{Conclusions}

Hydrogen bonding thermodynamically stabilizes $\beta$-sheets relative to polypeptide strands. Our results confer that the anti-parallel $14_{3} 10_{2} \beta$-sheet configuration to be more stable than the paralleland anti-parallel $10_{3} 14_{2}$ b-sheet configurations when no disulfide bridges are present. Introduction of disulfide bridges either within polypeptide strands (intra-polypeptide bridges) or betweenpolypeptide strands (inter-polypeptide bridges) results in b-sheetstabilization. The study found that upon introduction of either inter- or intra-strand disulfide bridges, the partially and fullyoxidized parallel pentapeptide $b$-sheets became more stable thanb-sheets in the $10_{3} 14_{2}$ and $14_{3} 10_{2}$ anti-parallel configurations. Inall cases, however, introduction of inter-strand bridges were more thermodynamically favorable than the introduction of intra-strand disulfide bridges. These results raise new questions in regards to the effect of disulfide bridge introduction within b-sheets due to the previous notion that if anti-parallel $b$-sheets were more stable than parallel $b$-sheets, that disulfide bridge introduction would only further stabilize anti-parallel structures in a similar manner compared to their parallel counterparts. It inspires one to speculate whether proteins with more parallel in 
comparison to anti-parallel b-sheets in their secondary structure are more at risk to overcome the kinetic barriers and become pathogenic-associated insoluble amyloid plaques. 


\section{References}

[1] C.A. Ross, M.A. Poirer, Nat. Med. 10 (2004) S10.

[2] D. Eisenberg, M. Jucker, Cell 148 (2012) 1188.

[3] M. Sunde, L.C. Serpell, M. Bartiam, P.E. Fraser, M.B. Pepys, C.C. Blake, J. Mol. Biol. 273 (3) (1997) 729.

[4] R. Nelson, M.R. Sawaya, M. Balbirnie, A.O. Madsen, C. Riekel, R. Grothe, D. Eisenberg, Nature 435 (7043) (2005) 773.

[5] A. Perczel, P. Hudaky, V.K. Palfi, J. Am. Chem. Soc. 129 (48) (2007) 14959.

[6] Y. Goto, H. Yagi, K. Yamaguchi, E. Chatani, T. Ban, Curr. Pharm. Des. 14 (30) (2008) 3205.

[7] G. Pohl, I. Jakli, I.G. Csizmadia, D. Papp, G.F. Matias, A. Perczel, Phys. Chem. Chem. Phys. 14 (2012) 1507.

[8] L.J. Perry, R. Wetzel, Protein Eng. 1 (1987) 101.

[9] S.D. Khare, E. Ding, N.V. Dokholyan, J. Mol. Biol. 334 (2003) 515.

[10] H.W. Klafki, A.I. Pick, I. Pardowitz, T. Cole, L.A. Awni, H.U. Barnikol, et al., Biol. Chem. Hoppe-Seyler 374 (1993) 1117-1122.

[11] W. Swietnicki, M. Morillas, S.G. Chen, P. Gambetti, Biochemistry 39 (2000) 424.

[12] T. Zako, M. Sakono, N. Hashimoto, M. Ihara, M. Maeda, Biophys. J . 96 (2009) 3331.

[13] Y. Chen, N.V.A. Dokholyan, J. Mol. Biol. 354 (2005) 473.

[14] Y. Liang, S.V. Pingali, A.S. Jogalekar, J.P. Snyder, P. Thiyagarajan, D.G. Lynn, Biochemistry 27 (2008) 10018.

[15] C.K. Smith, L. Regan, Science 270 (1995) 980.

[16] M.A. Wouters, P.M.G. Curmi, Proteins Struct. Funct. Genet. 22 (1995) 119.

[17] E.G. Hutchinson, R.B. Sessions, J.M. Thornton, D.N. Woolfson, Protein Sci. 7 (1998) 2287.

[18] S.M. Zaremba, L.M. Gregoret, J. Mol. Biol. 291 (1999) 463.

[19] K. Chakraborty, S. Thakurela, S.P. Ravindra, S. Indu, S.S. Ali, R. Ramakrishnan, R. Varadarajan, Biochemistry 44 (2005) 14638-14646.

[20] J.S. Richardson, Adv. Protein Chem. 34 (1981) 167.

[21] J.M. Thorton, J. Mol. Biol. 151 (1981) 261.

[22] P.J. Hogg, Trends Biochem. Sci. 28 (2003) 21.

[23] M.A. Wouters, R.A. George, N.L. Haworth, Curr. Protein Pept. Sci. 8 (2007) 484495.

[24] O. Carugo, M. Cemazar, S. Zahariev, I. Hudáky, Z. Gáspári, A. Perczel, S. Pongor, Protein Eng. 16 (9) (2003) 637.

[25] I. Hudáky, Z. Gáspári, O. Carugo, M. Cemazar, S. Pongor, A. Perczel, Bioinformatics 55 (1) (2004) 152.

[26] K. Chakraborty, S. Thakurela, R.S. Prajapati, S. Indu, P.S.S. Ali, C. Ramakrishnan, V. Raghavan, Biochemistry 44 (2005) 14638.

[27] J.M. Mason, N. Gibbs, R.B. Sessions, A.R. Clakre, Biochemistry 41 (2002) 12093. [28] A. Perczel, Z. Gáspári, I.G. Csizmadia, J. Comput. Chem. 26 (11) (2005) 1155.

[29] M.J. Frisch et al., GAUSSIAN 09, Revision A.02, 2009, Gaussian Inc., Wallingford, CT, USA.

[30] M. Frenkel, K.N. Marsh, R.C. Wilhoit, G.J. Kabo, G.N. Roganov, Thermodynamics of Organic Compounds in the Gas State, Thermodynamics Research Center,

College Station, TX, 1994.

[31] F.C. Bernstein et al., J. Mol. Biol. 112 (1977) 535-542. 
[32] P. Carter, C.A. Andersen, B. Rost, Nucleic Acids Res. 31 (13) (2003) 3293. 


\section{Figures:}

A

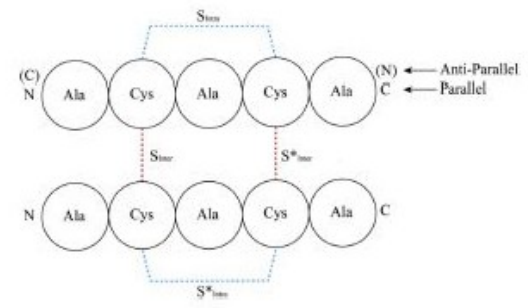

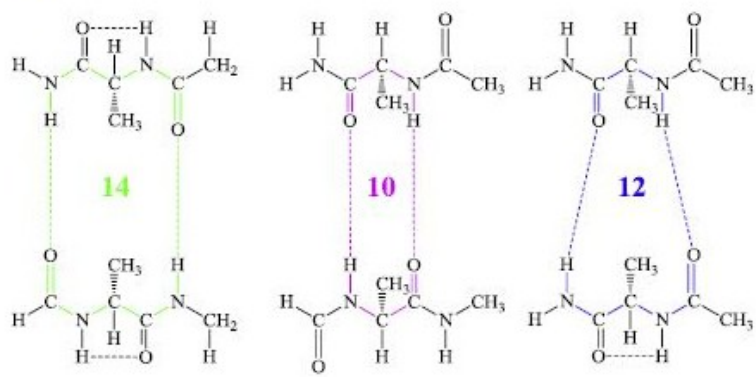

Figure 1. (A) Topology of the disulfide bridges studied; both anti-parallel and parallel arrangements as model systems were probed. Dashed lines indicate the two types of disulfide bridges, highlighting the intra- $\left(\mathrm{S}_{\text {Intra }}\right.$ and $\left.\mathrm{S}_{\text {Intra }}\right)$ and the inter-peptide $\left(\mathrm{S}_{\text {Inter }}\right.$ and $\mathrm{S}_{\text {Inter }}$ ) disulfide bonds between polypeptide chains. (B) Atomic structure of the alternating fourteen and tenmembered hydrogen-bonded rings used in the $14_{3} 10_{2}$ and $10_{3} 14_{2}$ anti-parallel models. The twelve-membered hydrogenbonded unit, appearing in parallel models is also depicted. (Dashed lines indicate H-bonding.)

A: Anti-Parallel: $14_{3} 10_{2}$
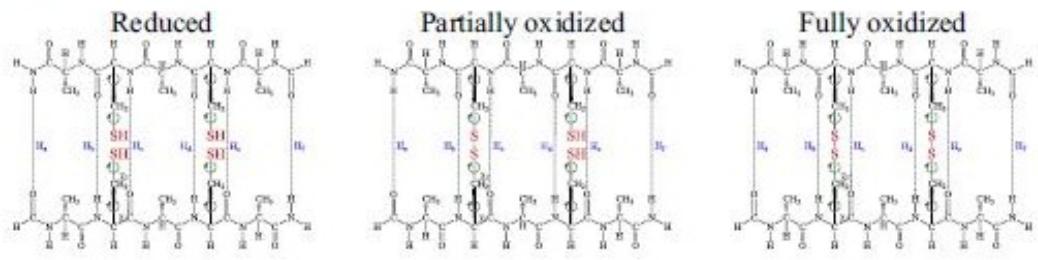

B: Anti-Parallel $10_{3} 14_{2}$
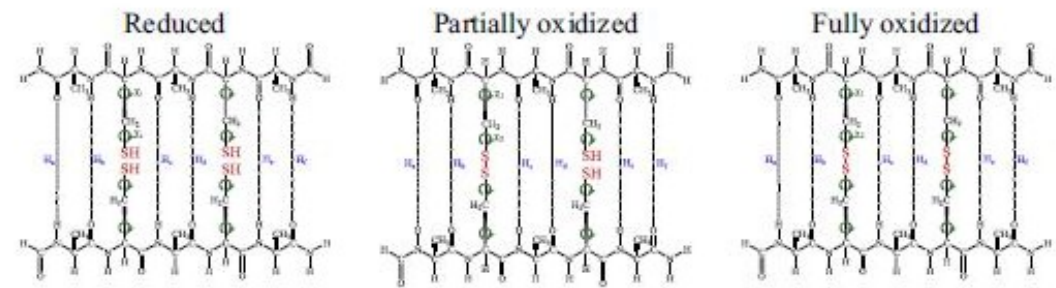

\section{C: Parallel}
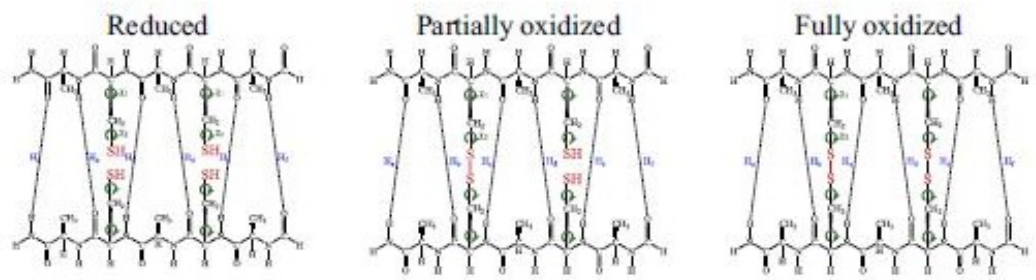

Figure 2. The reduced-, partially- and fully-oxidized forms of the (Ala-Cys-Ala-Cys-Ala)2 model systems all adopting extended like backbone structures (A) anti-parallel $14_{3} 10_{2}$. (B) Anti-parallel $10_{3} 14_{2}$ and (C) parallel main chain alignments. Schematic illustration of the dihedrals of interest $\chi_{1}$ and $\chi_{2}$ (curved arrows) and hydrogen bonds (dashed lines) are also provided. 


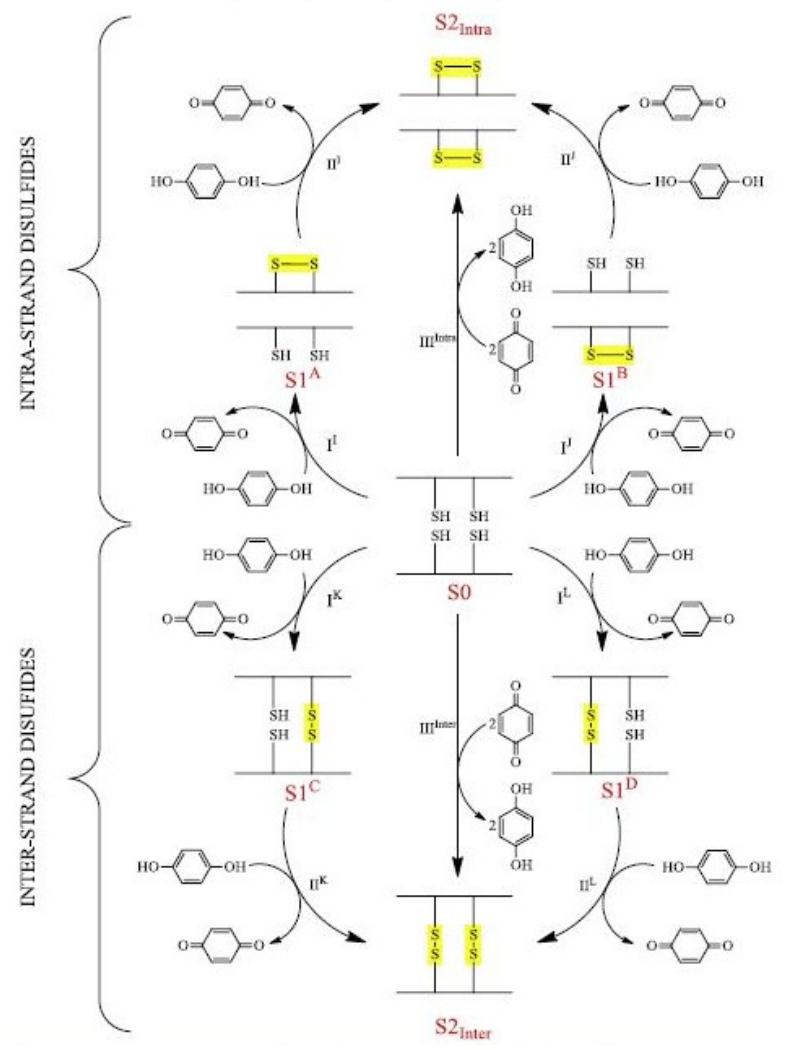

Figure 3. The thermodynamic cycle depicting the formation or reduction of two SS-bonds via three redox systems (I, II and III) for both intra and inter-polypeptide strand disulfides of (Ala-Cys-Ala-Cys-Ala)2 through the 1,4-benzoquinone oxidation pathway. Reaction pathways are labeled with roman numerals; reaction products/reactants are labeled as S0 (reduced), S1 (mono-oxidized) and S2 (di-oxidized) depending on the disulfide oxidation/reduction states of the cysteines within the pentapeptide. Alphabetic superscripts indicate the distinct mono-oxidation reaction pathways which the reduced (S0) pentapeptide may undergo depending on the location of the single bridge formation and its proximity to the pentapeptide's amino or carboxy termini.

A:

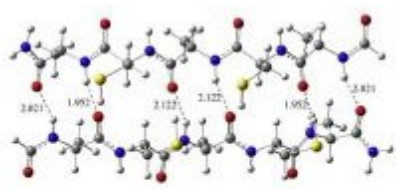

INTER

INTRA

INTRA

Lateral View

B:

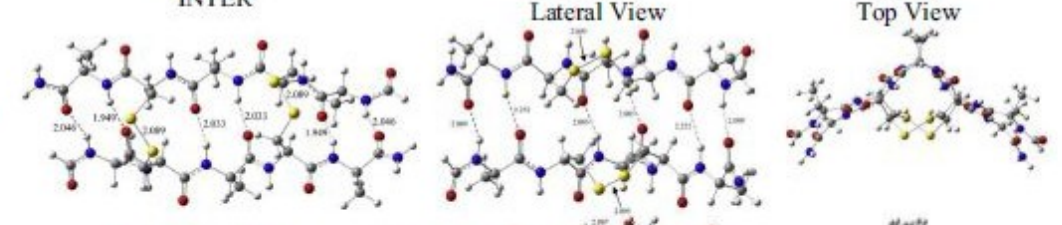

C:
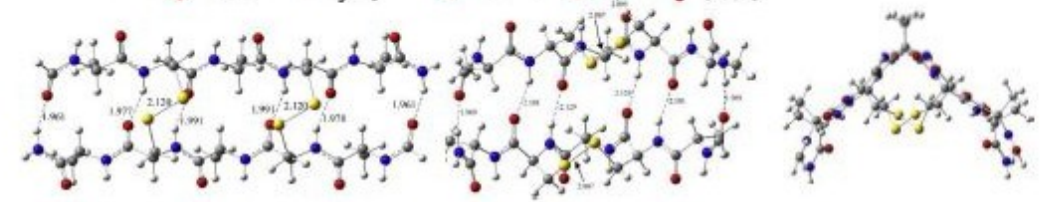

D:
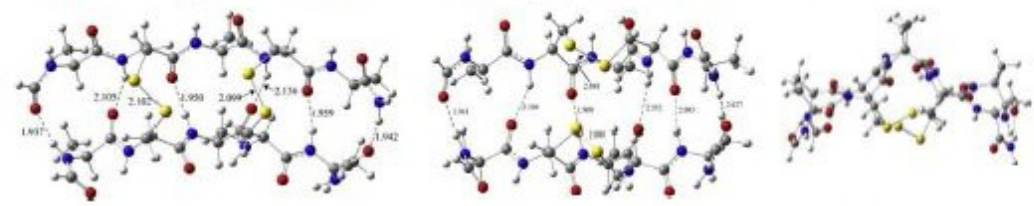
Figure 4. (A) The geometry optimized reduced form (4 CysSH) of $10_{3} 14_{2}$, with six backbone inter-stand H-bond lengths ( $\AA$ ) presented. (B-D) The fully oxidized (Inter and Intra) disulfide structures of $10_{3} 14_{2}(B) 14_{3} 10_{2}(C)$ and of the parallel model (D), with the same 6 H-bond, plus the two SS bond lengths $(\AA)$ depicted. Data obtained at the B3LYP/6-31G(d) level.

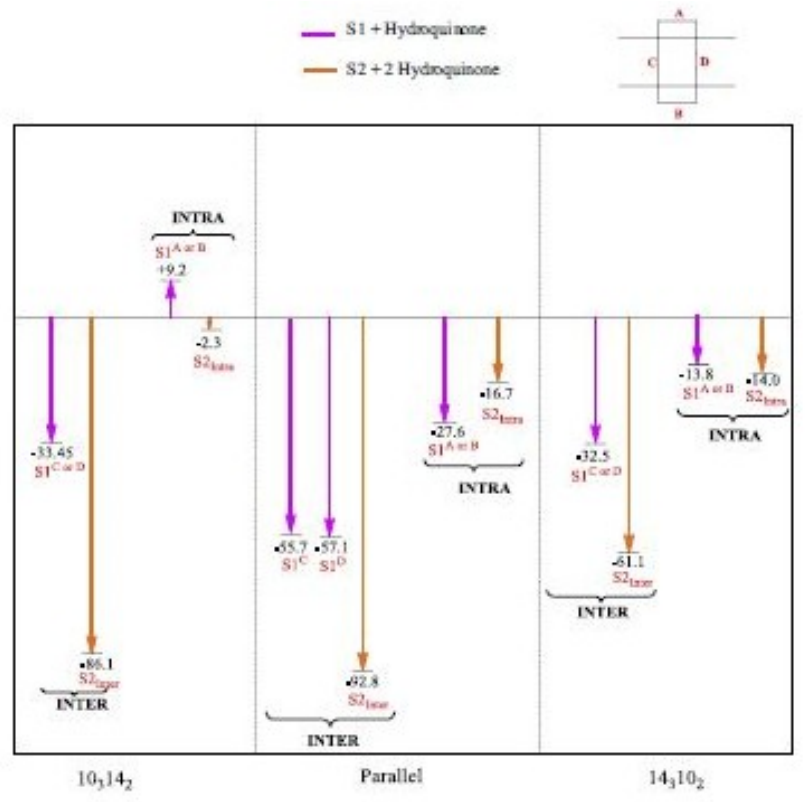

Figure 5. Schematic illustration of the relative free energy $\left(\Delta \mathrm{G}^{0}\right)$ differences $(\mathrm{kJ} / \mathrm{mol})$ between the single disulfide bridge (S1) and double disulfide bridge (S2) for all three models relative to a reduced Ala-Cys-Ala-Cys-Ala pentapeptide in b-sheet configuration (S0) (solid black horizontal line) for three disulfide formation reaction mechanisms using and 1,4benzoquinone oxidation. Alphabetic superscripts indicate the distinct mono-oxidation reaction pathways for which the reduced (S0) pentapeptide may undergo depending on the location of the single bridge formation and its proximity to the pentapeptide's amino or carboxy termini. Data obtained at the B3LYP/6-31G(d) level. 